\title{
Social aspects of resources management through process of understanding the social representations
}

\author{
Yana Vinogradova ${ }^{1,{ }^{*}}$, Svetlana Gurieva ${ }^{1}$, Ludmila Pochebut $^{1}$, and Vera Chiker $^{1}$ \\ ${ }^{1}$ Saint Petersburg State University, University Embankment, 7-9, 99034, Saint Petersburg, Russia
}

\begin{abstract}
The main use of our study is to highlight the concept of resource management through process of deep understanding the social representations in our society in different situation. The study traced the main differences in the concept and social representation of the phenomenon of betrayal in two age samples. Methodology: Projective methods are the main: semi-structured interviews, modification of the Sentence Completion Test. To obtain information on the actual attitude to the phenomenon we conduct the survey. The texts processed content analysis method. Selected differences in social perceptions in the studied groups of different ages reflect the boundaries of the concept. The analysis of structural components shows the dynamics of social representations. Emotional and rational styles of behaviour in a social situation, typical for a middle-age group, have been singled out. Behavioural styles differ in average values of "possibility of betray". Applications of this study: the results of the research are applicable both in training courses on the psychology of communication and in educational programs on forming a metacognitive assessment of the social situation. The show features of the actual attitude toward to social representations and possibility to analyse the main aspects of resources management.
\end{abstract}

\section{Introduction}

Trust and betrayal are the dynamic characteristic of relationships in modern society; it ensures and accompanies the process of integration and guarantees stability or variability, constantly or flexibility. Pressure, domination, demonstration of power and authority in order to regulate social processes has become the perfect rules in modern society. But pressure, sense of fear, injustice treason, exploitations on the workplace, discrimination, and demonstration crashed social trust-based relationships in the long term in our society [1]. So Trust becomes the central problem in modern word. Although lots of researchers are interested in understanding functions and mechanisms of trust [2]. Trust as a sociopsychological phenomena to be in the same plane with betrayal [3]. Betrayal as a socially significant situation is described in detail in the works of ancient Romans, in books of ancient Greek philosophers, as well as in modern literature. Great attention of authors

\footnotetext{
* Corresponding author: yana.e.vinogradova@gmail.com
} 
reflects essential interest to the phenomenon which could not be explained from rational positions of evolutionary theories of social development of humanity. Such characteristics make it possible to consider treachery as a stable social-psychological phenomenon, which implies similarity of social conceptions of the phenomenon in different groups, communities, cultures. The main aspects of the betrayal situation are similar for the authors of all times and different continents. Such stable notions of Betrayal provide an opportunity to study in detail its socio-psychological features in order to understand the links between human representations and behaviour in a social situation.

Socio-psychological phenomena arise as a result of interaction in open conditions and form the individual experience of the person. Phenomena are presented by people as events, cases or situations and form a series of life episodes. In this way, organizes the real knowledge of the subject about the regularities of interaction and the daily behaviour of individuals. Social knowledge and formed relations of the subject of interaction regulate his or her behaviour in groups and communities, as well as contribute to the organizations and regulation of social activity in a whole. In modern psychology, it is important to understand a person as active in constructing a situation based on objective conditions and personal features, without giving preference to either the individual or the situation in generalizing of social behaviour. In this approach to the determinism of behaviour, it becomes important to what a person directs his or her attention. In other words, people choose situations and create them for themselves and their goals [4]. In other words, there is a direct link between judging about Others and trust [5]. The scientific problem in this paper is related to the study of changes in a person's knowledge of the social situation, which contains all external and internal factors regulating interactions with the social environment. Notions of the social situation reflect the possibilities for actual social behaviour.

The theory of Moscovici is main perfect method to study social phenomena. The analysis of social perceptions leads to an understanding of the ways of transformation of modern social reality. Among the advantages of the approach are the universality of the proposed model researchers determined simultaneously by the subject himself (his history, life), the social and ideological system in which he act, and the nature of the subject's links to the system. Social representations condition the direction of a person's social behaviour and influence the entire process of his or her interaction.

\section{Literature review}

A social representation in psychology generally definite as mental education organizes into a system of values, ideas, and practices. Social representations allow us to understand the material and social world, establishing order in it and thereby subordinating ourselves [6]. By compiling a code, they allow to name and classify the objects of the world, uniting them into individual and group history, and provide communication of group members, which makes social exchange possible. The concept of social representations develops in school S. Moscovici since the early 1960s. The theory explains the mechanisms of false conceptions formation as an adaptation mechanism, formed under the influence of various facts of the surrounding social and natural world [7]. The works of S. Moscovici and his colleagues show the importance of social representations as mental education, reflecting the constant complication of mental processes. The field of research where the approach is applied includes the study of social phenomena and objects: transformations of established ones and the emergence of new ones in the conditions of modern society. A theory that emerged in S. Moscovici's dissertation research base on an analytical generalization of empirical material by extended methodological provisions, which determines the heuristic potential of the concept. Continuing the traditions of symbolic interactivity, Moscovici defines a set of representations as a symbolic universe of public life, changing in specific behavioural acts 
[8]. The basic concepts of theory: structure (core and periphery), functions and dynamics of ideas are the subject of social and psychological analysis of numerous studies. Modern researchers are unanimous in their opinion that the cognition of social representations of large groups forms their mental image [9]. Radina considers social representations as forms of social knowledge created by large groups, relevant in the context of practice and common sense (individual and group), aimed at communication, understanding and mastering the social environment $[10,11]$. They define them as an important regulator capable of leading to individually variable behaviour [12]. Emelianova notes that social representations reflect the emotional states of group [13]. The results, empirical studies of social representations, make up an actual knowledge about modern society, about the dynamics of processes [14] contained in the symbolic schemes of individual mental reality.

Dontsov \& Emelianova distinguish an excellent methodological style of research carried out in line with the Moskovichi's approach [8]. Application of field methods: unstructured conversation, analysis of opinions, content analysis of books and films, etc., fundamentally distinguishes the school of social representation. The method used allows registering actual changes in the social environment. Thus, form the knowledge of common sense, social representations are the base of people's behaviour in groups and communities, and also represent regulators of social activity. In Lerner's understanding, social representations are a filter of the perception of inconvenient situations and decoder them [15].

Betrayal concept is considered as a model of embarrassing situations. The studying of the phenomena of betrayal [16] provides an opportunity to study in detail its sociopsychological features to understand the links between social representations and behaviour in a social situation.

Nowadays, in psychology, the researches of betrayal phenomenon are presented by separate authors. The psychological significance of the betrayal phenomenon fully presents in Rahman's work. The research bases on determining the psychological significance of betrayal situations. The author identifies typical situations of Betrayal: infidelity, disloyalty, disclosure of confidential information, dishonesty and broken expectations of support when such support was needed. Rachman stresses a betrayal situation becomes a non-normative disaster event for an individual [17]. The author highlights the reactions of the betrayer: anger, jamming, low self-esteem, guilt, punitive thoughts and a sense of disruption. He also notes that the experience of a traitorous event can provoke and exacerbate psychopathology.

The interrelationships between a person's interpretation of betrayal situations and a particular awareness of actual life events reflect in the concept of the trauma of Betrayal [18]. This concept reflects the regularities of the mechanism of psychic regulation in situations of disaster level. Gobin \& Freyd describe it as a "cheater-detector mechanism $[19,20]$ and define it as "traitor blindness". Such a response is a specific adaptive reaction to a traumatic event. It represents the suppression of traces of negative emotions that arise as a result of psychological or social effects such as insults, abuse, and violence [21]. A reaction is developed and recorded in a situation of interpersonal interaction with close people with whom a person has to continue communicating [22]. Thus "treacherous blindness" becomes the main style of interaction in interpersonal relations. Presented by the authors as a symptom-complex, the developed style of interpersonal interaction is interrelated with specific meaning and, or the circumstances of the interaction situation. The selected symptom-co complex includes negative emotions and negative evaluation reactions, forms moral responsibility, fear of consequences, and a sense of the experience of violence [19]. Thus, the negative experience of interaction determines the style of behaviour in the group. Bysova stresses the importance of a person's ability to cope with emotions and negative experience of interaction, is reflected in the mental health level of the participants of interaction [23]. 
The theories of moral judgment take a special place in understanding the phenomenon of Betrayal. Researchers discuss the psychological significance of the attitudes to Betrayal in assessing morally appropriate behaviour. Treachery judgments form schematic prototypes of help/benefit. By automatically activating cognitive concepts of harm, the rejected action "breaks the purity" of social interaction. The communicative act causes people to judge such actions as immoral. Theories of moral judgment clarify the perception of the "traitor" as a moral agent that harms patience and causes behavior to be constrained. The socially-expected traits associated with the understanding of Betrayal as a possible behaviour of an interaction partner. Such a general traits such as purity and loyalty become a priority in interpersonal relationships [24].

As noted by social theorists cultures often prosper only to the point that people can count benevolent actions [25]. The action always compares with a socio-typical behaviour. In the domestic psychological tradition, the phenomenon of Betrayal is regarded as a marker of a society's destructive culture. It appears interrelate with a system of values that are not accepted by both culture and the reference group. In a study conducted by Gurieva and Borisova Betrayal take place among the phenomena that reduce the level of trust in interaction [26]. To a certain extent, on the one hand, the idea of the need to create and maintain trust relations continues to develop; on the other hand, there is an acute need to describe, definite and methodological everything that can eradicate it, wreck it, obliterate it, annihilate it. Thus, gradually the authors come to the fact that there is a need to study the social phenomenon of Betrayal. There is no doubt that the theory of Betrayal can be successfully applied in social-psychological practices and affect a variety of spheres of human activity [27]. The formed experience of communication is transferred to many spheres of life, including the negotiation process, for example. The research has shown that the level of suspicion, hostility between the participants of groups increases in a situation of uncertainty, limited resources, and does not exclude the manifestation of extremely negative forms, including Betrayal of the group's interests [28].

So, Betrayal presents as a complex social-psychological phenomenon, which determines the openness of the process of interpersonal interaction [29]. The social phenomenon of Betrayal base on certain stages [30]. It allows it to be identified, diagnosed, explained, accepted or not accepted, adjusted. First of all, it is necessary to decode the socialpsychological factors responsible for the phenomenon [31]. Further, to interpret those [32] and explain the process of their emergence, trying to clarify what was behind them, or what goals could justify them. These will certainly that this will result in the fact that the decision made or not made, which will affect the decision making and the correction of behaviour in the future.

We stress the necessary of studying the notion of the phenomenon of Betrayal as a complex social phenomenon penetrating all spheres of human activity. Our research aims are to highlight the concept of Betrayal and to study the social representations of the phenomenon of Betrayal. The formed representations of the social situation and their relationship to the repertoire of social behaviour constitute the resources of the individual for active interaction in the situation. The ability to use these resources determines the possibilities for adaptation in a social environment. We formulate the following hypotheses to implement the purpose of the study:

- the concept of Betrayal stable knowledge, revealed in social representations;

- social representations of the betrayal phenomenon represent by different structural components (cognitive, emotional, conative);

- the revealed differences in social representations of the betrayal phenomenon determine the style of behaviour in the situation, which reflect in the actual attitude towards Betrayal - "possibility of betrayal". 


\section{Materials and methods}

To form a complete picture of the phenomenon of Betrayal, first of all, we were interested in the content of the concept of Betrayal. Secondly, how the concept of Betrayal relates to the social perceptions of the phenomenon of Betrayal. Thirdly, highlight the components (cognitive, cone and emotional) in the structure of social representations about Betrayal. Fourth, to present the actual attitude qualitatively to Betrayal, defined as "the possibility of betrayal". We used: semi-structured interviews, the "Unfinished Proposals" method, and respondents' questionnaires.

Semi-structured interviews were the first stage of the research. The interviews conduct individually to clarify the general meaning field and to collect betrayal judgments to construct "unfinished offers. The semi-structured interview method has a rigid structure, which allowed for a more accurate systematization of the data collected. The interview contained three questions. Question 1: What is Betrayal? Question 2: What does to Betrayal mean? During the interviewing, it became clear that the answers to the second question may vary: a) betrayal is not allowed; b) betrayal when; c) betrayal to. The third question of the interview depends on the respondent's second answer. The options were applied: "Betrayal it is ...?" or "Traitor it is ...?".

Then respondents filled in "Unfinished sentences" to highlight the associative field and social representations of Betrayal. The method allows obtaining information about the fragments of reality as they are perceived by the respondent themselves, without any a priori imposed schemes. The initial version of the method Sentence Completion Test (SSCT), Sacks, Levy, 1950, develops to study specific clusters of human attitudes or spheres of his life [33]. The method is based on the concept of projective method and the technique of verbal associations. The projections are further interpreted by the experimenter, who has a certain set of keys and categories of analysis. Based on the SachsLevy test, modifications regularly develop which allow investigating the attitude of people to this or that area in experimental-psychological practice. We have modified the methodology for research purposes; remove many scales for reasons of time saving. Based on the basis of which new proposals have been developed, combined in the scale "attitude towards betrayal". The main feature of the developed proposals is that they focus on a short response from the respondent, containing associations rather than reflections on the nature of the phenomenon. The assembled associations make it possible to accurately define the structure of social representations according to the methodology developed in the Verges approach [34]. According to our method, comparing the structure of social representations (core and peripheral zones) with cognitive, emotional, and conative components reveals the essence of possible behavioural options in a situation. The analyses of the results base on this model. Respondents completed the betrayal proposals based on the patterns of perception of the phenomenon highlighted in the interview, while not responding to their experience of interaction, but describing their perceptions, thoughts, feelings, and behaviours. Here is an example of an unfinished sentence 1: "Betrayal.....". This sentence brought together more than 50 associations to this notion. Also interesting are the answers to the sentence 5 "Your actions in a situation of betrayal...". The answers differ from: "anger and resentment", "frustration", "despair" or "think about behavior", "understand the other", "count on yourself" or "choose who you interact with", "do everything right", "do not communicate". The presented examples illustrate the variants of emotional, cognitive and connotative components in the social notion of Betrayal.

The survey of respondents has a purpose to collect socio-demographic characteristics and studying the actual attitude to Betrayal - "possibility of betrayal". We asked respondents to rate 31 statements about Betrayal on the scale of "fully agree (5)/fully disagree (1)". Researchers developed the "betrayal response" proposals in focus groups of 
questions [35]. We take it as the basis for the closed statements about Betrayal. The statements were translated and adapted for research purposes.

The texts of interviews and "Unfinished Proposals" were processed using the content analysis method. Then, consider unit with selected elements of the respondent's judgment. Expert evaluates the consistency of the selected components (cognitive, emotional, and connotative) of the structure of social representations of the phenomenon of Betrayal.

The data process in SPSS 21. Descriptive statistics, method of average determination, comparative analysis were used.

78 women aged 12 to 50 years (average age 30.5 years) attend in a study. Participants represented the following groups: adolescence 1 (12-16 years) - 25.64 per cent of the sample; adolescence 2 (17-21 years) - 25.64 per cent; mean age 1 (22-34 years) - 25.64 per cent and mean age 2 (35-50 years) - 23.1 per cent of the sample.) We use the age separation by B.G. Ananyev to form the sample [36]. All are received higher education or are studying according to age.

Present the materials, methods, survey, questionnaire etc. used for the study. Author should explain whether this study is experimental, or review study, or simulation-based or survey-based. Discuss software, and hardware's, used during study with their brand names. Mention all research conditions, assumptions, theories followed. This section should be easy enough for any reader to repeat the study under similar conditions.

For the quantitative study: Include Population and sample [criteria of population selection and method of sampling] materials/tests with description, variables, Nature of data, data collection techniques, data analysis method. Discuss software, hardware's used during the study with their brand names. This section should be easy enough for any reader to repeat the study under similar conditions.

For the qualitative study: Include methodological orientation [grounded theory, discourse analysis, ethnography, phenomenology, content analysis] Sampling [number of participant, Method of approach, where the data was collected] Data collection [interview, Audio visual, field notes] and data analysis [number of data coders, software, etc.]

\section{Main findings}

Based on the results of interviews conducted with respondents of different age groups, statements were collected that defined the scheme of the concept of betrayal. The selected schemes can be sum up and presented in the following way: "Betrayal is bad, evil. Betray by other people. It is possible to betray, but do not betray for different reasons. I will be offended for a long time and will not communicate with such people". We based on these scheme when modifying the methodology "Unfinished sentences. Based on the results of the content analysis of "unfinished proposals", the components (cognitive, emotional, and cone) in the social representation of the groups under study were singled out.

For teenagers (12-16 years old) the cognitive component is represented by statements: "he"; "person"; "friend"; emotional component: "bad", "can not be", cognitive component: "not to be friends". For young people (17-21 years old), the cognitive component is made up: "person", "betrayal", "you", "deception", "nobody", "shame"; emotional: "has no feelings", "unhappy", "broken", "strong", "betrayer", "confident"; conative: "betray", "believe", "act", "betray", "tell less". Presentation of respondents of the group average age 1 (22-34 years) contains the following elements in the cognitive component: "traitor", "treachery", "deception", "shame", "harm", "interest", "goal", "respect", "stupidity", "farewell"; emotional component: "insatiable", "sneaky", "necessary", "unintentional", "without conscience and feelings", "weak; broken", in the conative component: "betray", "betray", "betray and forgive", "do not appreciate", "do not think", "think well". The representations of the group average age 2 (35-50 years old) are revealed in the cognitive 
component ("betrayal", "friend", "violation", "loyalty", "Agreement", "choice", "conscience", "rules of the game", "benefit", "claims", "value of communication", "everyone", "means of achievement", "consequences", "necessity). In the emotional component: "true", "sudden", "unknown", "possible", "close", "strong", "disappointed", "rare", "foul", "hard". And in the conative component: "lost", "forgiven", "not to forget", "unable to cope", "know and do", "not to appreciate", "exclude from the circle of communication". The presented examples of cognitive, emotional, and connotative components reflect the significance of the ontogenesis experience of communication for the established attitude towards interaction.

The components define qualitative differences in a person's attitude towards negative phenomena. Judgments about the event of Betrayal represent established attitudes and relate to behaviours aimed at reducing tension in the interaction. It is significant that in adolescence the basis of social representations of Betrayal lies within the limits of "he is bad - he is not a friend". Later on, already in adolescence, there are dynamic changes in the structure of social representations: "betrayer - deception - no one - shame". Styles of the behaviour of respondents in this group differ little, according to their social experience. In average age, changes in social representations become less intense. Still, one can observe a greater substantial variation in the cognitive, emotional, and behavioural components of the structure of the social representation. Briefly, the social representations of this group present as "deception - treachery - shame - interest - rules of the game - claims consequences - goodbye". At the same time, behaviour styles also become more variable. It should note that out of the total number of answers of all respondents (1320), only 30 are defined by respondents as positive, while the rest (1290) have negative values.

Thus, the components of social representations mark by noticeable substantive changes, which are most different in the middle age group. Dynamic changes in the structure of social perceptions take place in the youth period (16-21 years), unlike the teenage period (12-16 years). Sharp changes observe in the average age group (21-35 years). To fully describe the concept of Betrayal, it is important to compare the structure of social representations of betrayal of respondents in groups average age one and average age two. The figure 1 shows the component structure of social perceptions of betrayal (cognitive, emotional, conational) of group respondents average age (average age one year (21-34 years) and average age two years (35-50 years):

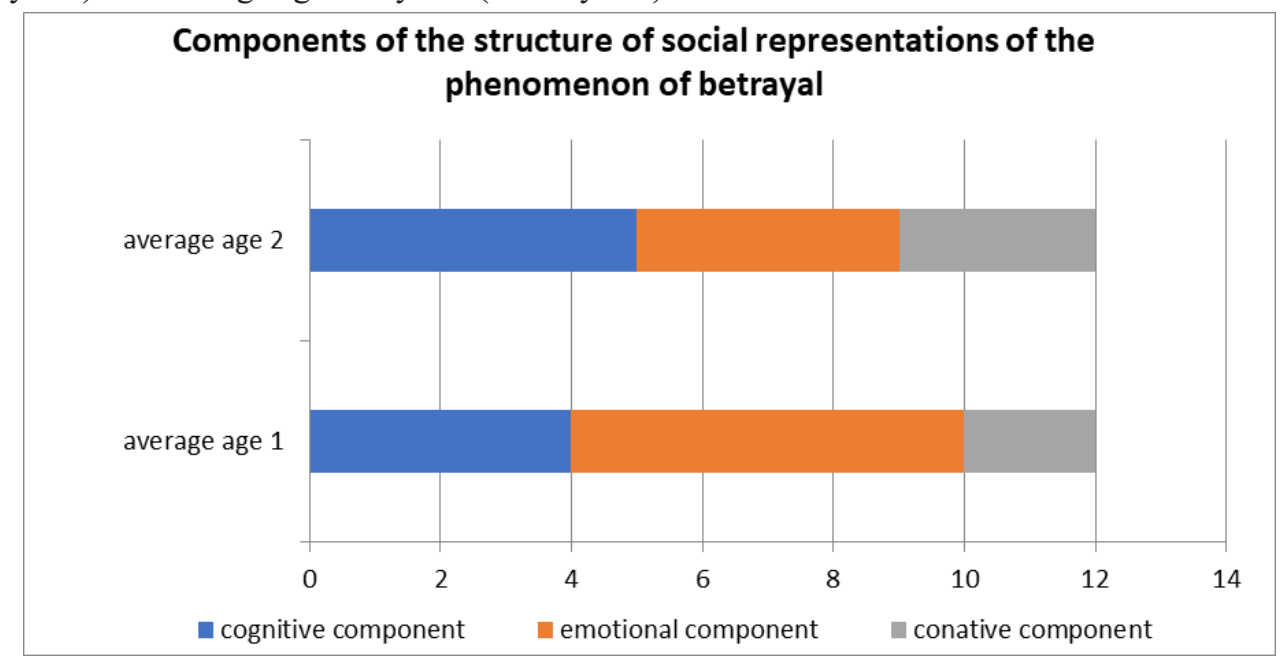

Fig. 1. Components of the structure of social perceptions of the phenomenon of Betrayal. 
The comparison of mean values of the components (cognitive, emotional, conative) in the groups mean age one and mean age 2 reflects qualitative changes in the concept of Betrayal while maintaining the boundaries without changes. Changes in the concept take place within the framework of cognitive and conative components in the respondents of group 2 average age (35-50 years old). The emotional component is noticeably wider in the respondents of group 1 (21-34 years old) compared to the respondents of group 2 (35-50 years old).

The highlighted variability is important for further, more detailed studies. At this stage, it can assume that young people's life experience allows them to understand Betrayal more easily, while for older respondents, betrayal becomes a less unequivocal social phenomenon. Group 1 (21-34 years old) respondents are differ by emotional experiences (resentment, anger, frustration, and confusion) when perceiving such situations, which can be considered a behavioural trait.

Respondents in group 2 (35-50 years old) stand out by more rational judgments and a wide range of opportunities for action in betrayal situations, which include: "lose", "forgive", "do not forget", "fail", "know and do", "appreciate", "exclude from the circle". The repertoire of actions possible in a situation of Betrayal, groups average age 1 (21-34 years) includes: "betray", "betray yourself", "not appreciate", "not think", "think well", "forgive and throw away". The singled out features of the concept of "betrayal" are not only the features of the social representations of the groups being compared, but also a variant of the behavioural style, a determinant of the behaviour of the individual as a whole.

According to the results of the analysis, the respondents after 31 years have high values of the actual attitude to Betrayal - "possibility of betray". We compare the results of the "possibility of betrayal" measurements in the groups. The obtained results present in the table 1:

Table 1. Comparison of mean values of components of the "betrayal structure" with mean values of "possibility of betrayal".

\begin{tabular}{|c|c|c|c|c|c|}
\hline & $\begin{array}{l}\text { The a } \\
\text { "poss }\end{array}$ & $\begin{array}{l}\text { age cost of the } \\
\text { ity of betrayal" }\end{array}$ & $\begin{array}{r}\text { The averag } \\
\text { re }\end{array}$ & $\begin{array}{l}\text { e of co } \\
\text { itations }\end{array}$ & $\begin{array}{l}\text { nents of social } \\
\text { cture }\end{array}$ \\
\hline \multirow{5}{*}{$\begin{array}{l}\text { The average } \\
\text { age of } 1 \\
\text { (21-34 years) }\end{array}$} & Mean & Std.Deviation & Components & Mean & Std.Deviation \\
\hline & \multirow{4}{*}{$\mathbf{5 1 , 9}$} & \multirow{4}{*}{11,9} & Cognitive & 3,7 & 1,8 \\
\hline & & & Emotional & 6,3 & 2,1 \\
\hline & & & Conative & 2,2 & 1,3 \\
\hline & & & $\begin{array}{l}\text { Overall } \\
\text { Value }\end{array}$ & 12,2 & 2,4 \\
\hline \multirow{4}{*}{$\begin{array}{l}\text { The average } \\
\text { age of } 2 \\
\text { ( } 35-50 \text { years) }\end{array}$} & Mean & Std.Deviation & Components & Mean & Std.Deviation \\
\hline & \multirow{3}{*}{39,2} & \multirow{3}{*}{6,0} & Cognitive & 5,3 & 1,6 \\
\hline & & & Emotional & 4,7 & 1,6 \\
\hline & & & $\begin{array}{l}\text { Overall } \\
\text { Value }\end{array}$ & 13,1 & 3,52 \\
\hline
\end{tabular}

The data presented in the table reveal the limits of the concept of Betrayal. We compare the average values of behavioral style variants (universal values of the components of the betrayal concept structures) as well as average values of "possibility to betray" differ in social conceptions of the groups. High values of "possibility to betray" (51.9) and emotional style of behavior (total value - 12.2: cognitive - 3.7; emotional - 6.3; conative 2.2) distinguish the social representations of the group average age of 1 (21-34 years). Representations of the group average age 2 (35-50 years) are characterized by lower average value of "possibility to betray" (39.2) and rational style of behavior (total value 13.1: cognitive - 5.3; emotional - 4.7; cone - 3.1). 


\section{Discussion}

The research aim is to study a person's ideas about the social situation. The actuality of the investigated problem is caused by a plurality of views to a problem of behavior determinacy. At actual nowadays this problem interprets as an interaction of personal and situational aspects. The aim concretizes in the study of the social situation of betrayal. The qualitative analysis of external and internal regulators of behavior in a situation compare with current opportunities in social interaction.

For a complete understanding of Betrayal, it was necessary first to understand the concept of Betrayal as a category that most fully reflects the basis of the differences in human behavior in similar social situations. The concept of what one knows thinks represents the objects of the external and internal worlds. It contains information about the social environment. This information refers to the actual or virtual perception of the world. A concept is a public image, a concept, a symbol in language, complicated by signs of individual representation. The concept has a complex structure, expressed by different groups of features, implemented in various linguistic ways and means - representatives of the concepts. The structure of a concept is a set of general features necessary and sufficient for the identification of an object or phenomenon as a fragment of a world picture. The information about an object (a fragment of the world) can be received by different ways of perception (using sight, hearing, smell, touch, etc.), and also can be selected into an opposition group in the structure of the concept. The result of research in the field of cognitive science was the statement about inextricable interrelation of mental processes. In the other words, the understanding of some new situation occurs through the search in the memory of a familiar situation most similar to the new one. "Concept-form is a two-stage process: perception (perceptual isolation of the object of cognition) and consciousness (giving the object of cognition meaning)" [37]. Investigators stress "that there is a relationship between self-concept and evaluation of product concept" and "suggests that the actual and ideal self-congruence have a variable influence "on that process [38].

Social representations are the basis for the determination of people's behavior in groups and communities, and also represent the regulators of social activity. Researchers of representations demonstrate the ways, by which knowledge and social practice develop from any socially significant object. Social representations are systems of communication and social influence that constitute the social realities of different groups in society [39]. In general, researchers define social concepts as a system of communication and social influence that constitute the social realities of different groups in society [40]. Thus, we assumed that social representations and concepts relate as parts and as a whole, reflecting possible patterns of behavior in a social situation. The structure of social representations singled out by S. Moscovichi: information, the field of representation, and attitudinal, is correlated with the structure of the concept. "Social representations are a key concept within social science" are studying "to shed light on the semantic field and cognitive organization of a given social representation" [41]. We have described in detail the structure of the social perception of the phenomenon of Betrayal [42]. Different changes of components inside the borders of the concept of betrayal show the phenomenon of cognitive polyphagia, which is typical for the reflection of complex social and psychological phenomena. The revealed differences in the repertoire of behavior offered by the respondents in the situation have raised the question of relations of social representations and attitudes. According to Moscovichi, attitude is a ready-made behavioral scheme. The question of relations between social attitudes and the notion of "social representations" has been discussed by researchers on several occasions. "Attitudinal competence is defined as a state of readiness to react to an object in a certain way as a form of evaluation and reflection of feeling" [43]. "The European approach of social 
representation is a means for socializing these concepts. "Social representation focus on community, collective practices and the institutionalization of social knowledge" [44]. It notes that the differences identified between social representations and social attitudes do not clarify the differences in the components of attitudes and structures of social representation. To obtain such data in our study, we distinguish components and compare them by differentiating group views. The results of the study of social representations of teenagers (12-16 years old), young men (17-21 years old), and middle ages show possible variants of the width of the concept of Betrayal. Components (emotional, cognitive, and conational) of the attitude explain the differences in the possibility of actions in a situation. For example, for a group of teenagers, the possibilities of action are limited to "betrayal bad - not to be friends". Such representation must be characteristic for a part of respondents in groups of young men and average ages 1 and 2. A focus on breaking social interaction characterizes such type of representation. The variants of the levels of the concept of Betrayal obtained in the research (cognitive, emotional, and conational), singled out from the social representations of the phenomenon by the respondents of different age groups, represent the variants of negative attitude increasing the level of distrust between people. Such attitudes, schemes contain markers of distrust. At the same time, only a small part of respondents suggest constructive ways of dealing with the social situation. Negative attitudes limit the social resources of co-operating and are completely solved at the expense of personal resources, being limited more often to the experience of negative emotions. Behavior styles represent different types of coping behavior: aimed at changing the situation or at adapting to it. The first one we describe as a change of situation where what is happening is controllable. In contrast, the second one is characterized by loss of control over the situation, which does not allow changing it [45]. So, is mistrust is a level of control? The functions of distrust are self-preservation and isolation. In this case, the main functions of trust (cognition, exchange, and provision of interaction) violate. In that model phenomena of trust and distrust are definite as independent of each other. And the model also includes three components: cognitive, affective, and behavioral [46]. Gurieva and Borisova investigated the phenomenon of trust, according to the model of Kuprechenko A.B., and described a set of ideas about trust, corresponding to a certain age stage [26].

It is important to compare the attitude of trust with the attitude of distrust in the context of Betrayal. Dunning et al. show the meaning of distrust during the first acquaintance and highlight the foundations of trust, which depend more on the learned norms than on any consistent calculations of the results of the interaction [47]. Authors mention that "trust is a paradoxical phenomenon". The authors' position about the nature of trust is "depend crucially on a disconnection between risk tolerance and social expectations on one side of the ledger and trust behavior on the other" [47]. The three types of behavior investigated in the experiments [48] and subsequently described are those that highlight trust and mistrust [49]. Investigators underline that "this is necessary for trust to fulfill its vital role in intimate relationships, friendships, organizations, and societies as a whole [48]. Simple trust or "Trust as an ordinary decision" [47] coincides with distrust of a stranger. Principled trust [50] can be briefly described as "trust out of courtesy" and described as socially desirable behavior guided by social emotions and feelings. The third type of behavior is "aversion to betrayal" [51]. It is "sygrophobia" or fear of exploitation based on attempts not to make oneself vulnerable to the doubtful reliability of an interaction partner. "Especially among economists, the concept of betrayal aversion has become quite ubiquitous as an explanation for (dis)trust behavior" [52]. "Sygrophobia" or "principled distrust", implies trust under unknown conditions and distrust under known conditions, and most fully reflects the frequently encountered behavior in our sample. It is necessary to add that we also singled out one more type of behavior that is more common in older age groups. This type of behavior can be defined as "knowing and doing". The decision to trust this type of behavior 
is based on comparison with other people: "Trusted", "Doing", "Betraying", "Cheating". This type of behavior differs from "trust out of courtesy" when the decision to trust reduce due to negative emotions. It also differs from "simple trust" because this type of behavior depends more on learned norms than on comparison with other people. The above types are part of the social representations of the study group and to some extent, determine the openness or closeness of interaction intense social situations. Thus, the expression of this or that type of behaviour determines the style of behavior of the group. Measurements of the group's behavioral style are of great prognostic value for studies of the social-psychological climate. Determining how cultures develop and then support cooperative actions among strangers is central to how people create interpersonal social norms and orders that are slightly different far from civilized, coercive, and crude measures. Cultures often flourish only to the extent that people can rely on the benevolent actions of an array of strangers. The phenomenon of Betrayal as a spectrum of negative situations represents a sociopsychological factor in the destruction of social capital, both at the personal and societal levels. The social and psychological climate of the organization is interrelated with the main style of the group. According to researches Pochebut et al., the psychological climate is one of the interrelated factors of a predictive model of trust in the organization [53].

\section{Conclusion}

So, this research suggests that perceptions of the situation form an opportunity to understand and act actively in a situation of social interaction. The variability of behavior strategies is also related to social representations of the situation since strategies largely determine the norms and procedures adopted in the group. Representations of a social situation, as a resource of the individual, allow different interactions in both everyday and complex social situations. Hypotheses of the study showed complex aspects of the phenomenon under study. To sum up, the concept of Betrayal change through social representations of different age groups. Representations change through the expansion of components (cognitive, emotional, conative) of the concept. The main differences observed are the tendency to expand the cognitive component about Betrayal. In this case, the boundaries of the emotional component narrow, and the behavioral component expands, while the negative attitude towards betrayal decreases (changes into ambivalent). The selected changes are presented below in general schemes. "Betrayal is bad" for respondents aged 12-16 years. "Betrayal is cheating" and "Betrayal is harm" for respondents aged 1721. "Betrayal for reasons" for respondents aged 22-34 years. Perceptions of the phenomenon in the group average age 2 (35-50 years) differ by a wide variety of cognitive components, includes all of the above schemes, and supplement by the scheme "Betrayal the choice of another person". We emphasize that the perceptions of the 12-16-year-old group remain the same for some respondents in the middle age group. The described schemes, which are part of the concept of Betrayal, represent established attitudes and include the behavioral styles aimed at reducing tension in the interaction. Strategies present in three main ways: stop communicating, form contacts, and change attitudes towards an interaction partner.

The researched actual attitude to Betrayal, "possibility of betrayal", shows high expectations of Betrayal with a high value of the emotional component and is designated as an emotional response style. Low values of "opportunity to betray" correlate with a high value of the cognitive component and designate as a rational response style.

The study also determined that the concept of Betrayal includes a wide range of negative situations, cases, events, and reactions, styles, and behavioral strategies. The structure of the social representation of Betrayal shows the social significance of the betrayal phenomenon, which reflects the mechanisms for changing the direction of 
interaction within a group, collective, and community. Presentations that lack formed strategies to assess negative aspects of the social situation determine changes in behavior in the direction "from people" and become the basis for fixing the negative assessment of the interaction partner. The component structure of social representation explains the process of forming a metacognitive evaluation of the social situation. The components of the social representations of compared age groups present general features: purity (average age 1) or loyalty (average age 2). The prevalence of one of the above components will determine the level of regulation of behavior and stereotypical knowledge of common sense. The components reflect the essence of the regulatory function of social representations in everyday interaction. The two-stage concept process involves different levels of perceptions of the social situation. The levels include the above types of behavior and characterize by increased mistrust and decreased trust, respectively, and reflect in actual strategies of interaction in the social situation.

Notions of distrust as a resource allow identifying the functions of distrust in the social situation that are not limited to self-preservation and isolation. The attitude of distrust determines the alertness of partners in interaction and only in case of unfavorable development of the situation leads to the termination of interaction and limitation of the number of social contacts.

The developed questionnaire allows us to measure the "possibility of betrayal", to determine the attitude to the social situation, as well as to determine how a person will perceive interpersonal contacts. A low "possibility of betrayal" differs by the variability of behavior in situations, which name as Betrayal. Expressed emotional reactions to complex social phenomena, situations defined as similar to Betrayal, are a marker of high psychosomatic behavior, and high "possibility of betrayal".

\section{Limitation and study forward}

In summary, this research suggests the importance to understand the social-psychological aspects of the phenomenon of Betrayal. The results of this study limit by the peculiarities of the object of research. Investigating of phenomena of betrayal in different ages, professional groups and communities will be useful as in different cultures as well.

Further investigations are possible using additional methodological techniques in the following main areas. First, the formed emotional assessment, understood as an attitude represents a special attitude to the social situation, as well as an aspect of the perception of the context of interpersonal interaction. But it remains unclear what can be the basis for changing the formed type of behavior: changing the breadth of the cognitive component, changing the variability of useful strategies, or changing a negative attitude to an ambivalent one. The types of behavior highlighted in the study require more detailed research.

Second, what is the actual reflection of the situation and how such evaluation differs from the metacognitive understanding of the situation, which is necessary for making the right decision? What makes it possible to have the necessary resources for that? The differences in the concept of situations, both everyday and difficult interaction situations, included by the respondents, suggest different ways of reading the context of the situation. It is also unclear how an individual response style is shaped that differs from the social view of the group.

Thirdly, it is important to study both the individual style to act in negative situations and the peculiarities of forming the group's style of behavior in such situations. The previous researchers show that the event of Betrayal is a marker of unfavorable intra-group relations. But it is not clear what can contribute to constructive changes in such group situations. The peculiarities of the concept of betrayal, registered in the group, the organizations, society, 
are presented as a socio-psychological factor of destruction of the social capital of an individual and the organization level. The study of distrust as a resource in situations of interaction is important for further understanding of its significance for group interactions.

We would appreciate receiving feedback and comments on the paper from its readers. We are grateful to our unknown reviewers for their review and comments that greatly improved our manuscript. They will help us complete and enhance research.

This research work was supported and funded by the Russian Humanitarian Science Foundation (RHSF) project № 19-013-00560 A.

\section{References}

1. B.C. Rathbun, Internat. Theory 1, 3, 345-380 (2009) https://doi.org/10.1017/S1752971909990121

2. P.J. Zak, S. Knack, The Econom. J. 111, 295-321 (2001) https://doi.org/10.1111/1468$\underline{0297.00609}$

3. R. Cubitt, S. Gächter, S. Quercia, J. of Econom. Behav. and Organiz. 141, 110-121 (2017). https://doi.org/10.1016/j.jebo.2017.06.013

4. E.P. Belinskaya, Social psychology of personality (Academia Publishing Centre, 2009)

5. M. Watabe, H. Ban, H. Yamamoto, Letters on Evolution. Behav. Sci. 2, 28-32 (2011) https://doi.org/10.5178/lebs.2011.16

6. W. Wagner, R. Farr, S. Jovchelovitch, F. Lorenzi-Cioldi, et.al. Asian J. of Soc. Psych. 2, 95-125 (1999) https://doi.org/10.1111/1467-839X.00028

7. S. Moscovici, Euro. J. of Soc. Psy. 18, 211-250 (1988)

8. A.I. Dontsov, T.P. Emelianova, The concept of social perceptions in modern (French psychology. Moscow University, 1987)

9. I. Marcova, Cadernos de Pesquisa 47, 163, 358-374 (2017) https://doi.org/10.1590/198053143760

10. M.G. Alexander, S. Levin, P.J. Henry, Politic. psy. 26, 1, 27-45 (2005) https://doi.org/10.1111/j.1467-9221.2005.00408.x

11. N.K. Radina, Penza psy. bulletin, 34-59 (2016) https://doi.org/10.17689/psy-2016.2.4

12. S.E. Serrano, Acta Colombiana de Psicología 16, 2, 63-70 (2013) https://doi.org/10.1590/19805314376010.41718/ACP.2013.16.2.6

13. T.P. Emelianova, Knowledge. Understanding. Skill 1, 213-223 (2015) http://doi.org/10.17805/zpu.2015.1.20

14. J. Wrange, R. Bengtsson, Euro. Security 28, 4, 449-472 (2019) https://doi.org/10.1080/09662839.2019.1665517

15. M.J. Lerner, D.T. Miller, Psy. Bull. 85, 1030-1051 (1978)

16. R. Zeckhauser, I. Bohnet, J. of Econ. Behav. \& Organiz. 55, 4, 467-484 (2004) DOI: $10.2139 /$ ssrn.447660

17. S. Rachman, Behav. Res. and Therapy. 48, 4, 304-11 (2010) https://doi.org/10.1016/j.brat.2009.12.002

18. Sh. Bach, The Int. J. of Relational Perspectives 28, 5, 557-568 (2018) https://doi.org/10.1080/10481885.2018.1506214

19. R.L. Gobin, J. Freyd, Psy. Trauma: Theory, Research, Practice, and Policy 1, 3, 242-257 (2009) https://doi.org/10.1037/a0017469 
20. J. Van Lier, R. Revlin, W. De Neys, PLoS ONE 8, 1 (2013) https://dx.doi.org/10.1371\%2Fjournal.pone.0053827

21. M.G. Platt, J.J. Freyd, Psy. Trauma: Theory, Research, Practice, and Policy 7, 4, 398 404 (2015) https://doi.org/10.1037/tra0000022

22. B.C. Delker, J.J. Freyd, J. of Aggression, Maltreatment, \& Trauma 26, 701-716 (2017) https://doi.org/10.1080/10926771.2017.1308982

23. V.M. Bysova, Novosibirsk State Pedagogical Univ. Bulletin 8, 5, 41-56 (2018) http://dx.doi.org/10.15293/2226-3365.1805.03

24. D. Selterman, A.C. Moord, S. Koleva, Person. Relationships 25(1), 65-68 (2018) https://doi.org/10.1111/pere.12228

25. E. Fehr, U. Fischbacher, S. Gächter, Human Nature 13, 1-25 (2002) http://doi.org/10.1007/s12110-002-1012-7

26. S.D. Gurieva, M.M. Borisova, Modern Res. of Soc. Problems 8, 2, 20-39 (2017) https://doi.org/10.12731/2218-7405-2017-2-20-39

27. S.D. Gurieva, M.M. Borisova, Modern Res. of Soc. Problems 12, 68, 138-150 (2016) https://doi.org/10.12731/2218-7405-2016-12-138-150

28. S.D. Gurieva, U.A. Udavikhina, Mediterranean Journal of Social Sciences 6, 4, 2, 109 117 (2015) https://doi.org/10.5901/mjss.2015.v6n4s2p

29. J. Henrich, Science 312, 60 - 61 (2006) https://doi.org/10.1126/science.1126398

30. B.M. DePaulo, K.L. Bell, J. of Personality and Soc. Psy. 71, 703-716 (1996) https://doi.org/10.1037/0022-3514.71.4.703

31. O. Stavrova, D. Ehlebracht, J. of Personality and Soc. Psy. 110, 116-132 (2016) https://doi.org/10.1037/pspp0000050

32. D. Fetchenhauer, D. Dunning, J. of Econ. Behav. \& Organiz. 81, 2, 534-541 (2012) .https://doi.org/10.1016/j.jebo.2011.07.017

33. A.P. Pahomov, Experim. Psy. 5, 4, 99116 (2012)

34. G. Lo Monaco, A. Piermatteo, P. Rateau, J.L. Tavani, J. for the Theory of Soc. Behav. 47(3), 1-26 (2017) https://doi.org/10.1111/jtsb.12124

35. K. Komolafe, Doctoral Clinical Psychological Thesis 1, 190 (2016)

36. B.G. Ananyev, Pedagogy 2(1), 89-95 (1980)

37. M.V. Pimenova, Bulletin of KemGu 2, 2, 127131 (2013)

38. Y. Ekinci, M. Riley, J. of Retailing and Consumer Services 10, 4, 201-214 (2003) DOI: $10.1016 / \mathrm{S} 0969-6989(02) 00008-5$

39. G. Sammut, C. Howarth, In: Teo T. (e) Encyclopedia of Critical Psychology, Springer, New York, NY (2014) https://doi.org/10.1007/978-1-4614-5583-7

40. P. Rateau, P. Moliner, Ch. Guimelli, J-Cl. Abric, Handbook of Theories of Soc. Psy. 2 , 477-497 (2012) https://doi.org/10.4135/9781446249222.n50

41. I. Tsoukalas, Qual Quant 40, 959-981 (2006) https://doi.org/10.1007/s11135-0055077-3

42. S.D. Gurieva, Y.E. Vinogradova, Herald of Vyatka State U. 3, 133, 132-139 (2019) https://doi.org/10.25730/VSU.7606.19.044

43. S' at A. Hudha, D. Mardapi, Research and Evaluation in Edu. 4(1), 35-44 (2018) DOI: https://doi.org/10.21831/reid.v4i1.20304

44. A.F. Bidjari, Procedia - Soc. and Behav. Sci. 30, 1593-1597 (2011) https://doi.org/10.1016/j.sbspro.2011.10.309 
45. N.M. Borisova, I.V. Shapovalenko, Psychological-Educational Studies 10, 3, 115-125 (2018) https://doi.org/10.17759/psyedu.2018100310

46. A.B. Kupreichenko, PFUR Newsletter, Psy. and Pedag. Series 2, 46-53 (2008)

47. D. Dunning, J.E. Anderson, Th. Schlösser, D. Ehlebracht, D. Fetchenhauer, J. of Personality and Social Psy. 107, 1, 122-141 (2014) http://dx.doi.org/10.1037/a0036673

48. D. Fetchenhauer, D. Dunning, J. of Econ. Psy. 30, 263-276 (2009) https://doi.org/10.1016/j.joep.2008.04.006

49. D. Dunning, D. Fetchenhauer, T. Schlösser, Current Directions in Psy. Sci. 28, 4, 366371 (2019) https://doi.org/10.1177/0963721419838255

50. D. Lerman, J. of Serv. Marketing 20, 92-100 (2006) https://doi.org/10.1108/08876040610657020

51. J.A. Aimone, S. Ball, B. King-Casas, PLoS ONE 10, 9, e0137491 (2015) https://doi.org/10.1371/journal.pone. 0137491

52. D. Fetchenhauer, A-S. Lang, D. Ehlebracht, T. Schlösser, D. Dunning, J. of Behav. Decision Making, 1-11 (2019) https://doi.org/10.1002/bdm.2166

53. L.G. Pochebut, S.D. Gurieva, V. Chiker, A. Soc. psy. and society 9, 1, 2242 (2018) https://doi.org/10.17759/sps.2018090103 